\title{
Spirally polarized beams for polarimetry measurements of deterministic and homogeneous samples.
}

\author{
Juan Carlos González de Sande ${ }^{\mathrm{a}, *}$, Massimo Santarsiero ${ }^{\mathrm{b}}$, Gemma Piquero $^{\mathrm{c}}$ \\ ${ }^{a}$ Departamento de Teoría de la Señal y Comunicaciones, Universidad Politécnica de \\ Madrid, Campos Sur, 28031 Madrid, Spain \\ ${ }^{b}$ Dipartimento di Ingegneria, Universitá Roma Tre, Rome, Italy \\ ${ }^{c}$ Departamento de Óptica, Universidad Complutense de Madrid, 28040 Madrid, Spain
}

\begin{abstract}
The use of spirally polarized beams $(\mathrm{SPBs})$ in polarimetric measurements of homogeneous and deterministic samples is proposed. Since across any transverse plane such beams present all possible linearly polarized states at once, the complete Mueller matrix of deterministic samples can be recovered with a reduced number of measurements and small errors. Furthermore, SPBs present the same polarization pattern across any transverse plane during propagation, and the same happens for the field propagated after the sample, so that both the sample plane and the plane where the polarization of the field is measured can be chosen at will. Experimental results are presented for the particular case of an azimuthally polarized beam and samples consisting of rotated retardation plates and linear polarizers.
\end{abstract}

Keywords: Polarimetry, Polarization,

\section{Introduction}

Knowledge of the optical characteristics of materials is of great importance for a large number of applications, ranging from design and fabrication of photonic devices to noninvasive biomedical diagnostic techniques (see, for 5 example, [1-3]).

Optical characterization of materials generally involves polarimetric measurements [4-8]. Using the Stokes formalism [9], any polarization state can

\footnotetext{
${ }^{*}$ Corresponding author

Email address: jcgsande@ics.upm.es (Juan Carlos González de Sande)
} 
be represented by a (four-element) Stokes vector. The effect of a sample on the polarization state of the light passing through it can be described by a $4 \times 4$ real matrix, known as Mueller matrix, which completely characterizes the polarimetric properties of the material [4-6]. In general, in order to get all the elements of the Mueller matrix, different states of polarization of the incident light have to be used, to complete a set of 16 measurements [4-6]. Such states are customary obtained by using polarizers and phase plates act15 ing on an incident field. Some methods, based on polarization modulation, use rotating anisotropic optical elements to produce a relatively large set of incident polarization states in sequence and recover the sample characteristics by means of signal analysis [4, 6, 10]. Recently, two Simon-Mukunda gadgets [11, 12 have been experimentally used to sequentially generate four different polarization states and to analyze the exiting light [13. In divisionof-amplitude polarimetry, to determine the state of polarization of the light exiting the sample, the output beam is split into four, and the replicas are simultaneously sent onto four analyzers [4, 6, 14, 15]. When used to recover the optical properties of a sample, all these methods make use of a totally and uniformly polarized incident light.

Several techniques have been proposed to improve the polarization state generator, the analyzer, or both [11, 13, 16 27]. Some of them are faster or easier to apply and others do not require many changes in the experimental arrangement, so that potential problems, such as misalignments, are reduced. In particular, the use of suitably designed beams presenting a nonuniform distribution of the polarization state across their transverse profile has been proposed [21, 22]. If states of polarization represented by four linearly independent Stokes vectors are present in the beam cross section, the Mueller matrix of a sample can be obtained from a relatively small number of meameasurements, the polarization distribution across the section of the incident light has to be carefully imaged onto the sample, because the polarization pattern changes after propagation and may also be altered by the imaging system.

40 Examples of nonuniformly polarized beams for which the evolution in propagation of their polarization pattern is known can be found elsewhere for completely coherent beams [28 32] as well as for partially coherent beams [30, 33, 36].

Recently, the use of a radially polarized input beam has been theoretically proposed for single-shot real-time polarimetry [26]. In particular, the authors 
propose the use of the entanglement of polarization and spatial degree of freedom of vector beams for the determination of sample Mueller matrix involving minimum number of measurements. A shortcut of the method is the necessity of using a large number of amplitude divisors which, as the thors recognize, could compromise its implementation.

In this paper, we propose the use of spirally polarized beams (SPBs) [37, 38 for polarimetric measurements of homogeneous, deterministic and transparent samples. SPBs present a circularly symmetric field profile and linear, but non-uniform polarization across the transverse plane. In particular, the polarization state is axially symmetric and the electric field lines are logarithmic spirals. SPBs include, as limiting cases, radially and azimuthally polarized beams. Up to now, beams of these types have been shown to be useful in applications, such as microscopy, material processing, manipulation of atoms or molecules (see Ref. [39] and references therein).

Two features of SPBs are particular appealing for their application in polarimetry. First, across any transverse plane all possible linear polarization states are simultaneously present and, second, the transverse polarization pattern is invariant upon propagation [37, 40. In fact, as it will be explained in more detail later, dealing with homogeneous and deterministic 41 samples, it will be sufficient to measure the first three Stokes parameters of the beam emerging from the sample at a set of three different points across a transverse plane to recover the complete Mueller matrix of the sample. If the intensity values necessary to the evaluation of the Stokes parameters of the beam are detected by a CCD camera, as in the experiment we are going to present, the data corresponding to all the points of the transverse plane are simultaneously acquired, so that a huge number of point sets will be available at once. This can be used to reduce the experimental measurement standard deviation values so a higher accuracy of the experimental measurements is expected. Furthermore, due to the propagation invariance of the polarization pattern of the output beam, the transverse plane where to acquire the intensity profiles can be chosen at will.

The paper is structured as follows: in Sec. 2 the formalism is introduced, together with the numerical approach used to recover the Mueller matrix of the sample. Spirally polarized beams are recalled in Sec. 3, while Sec. 4 describes the proposed experimental technique. Upper bounds for the errors in the determination of the matrix elements and optimum conditions for obtaining them are given in Sec. 5. Experimental results, pertinent to the particular case of an azimuthally polarized beam and samples consisting 
of rotated retardation plates and linear polarizers, are reported in Sec. 6 .

Conclusions are given in Sec. 7 .

\section{Preliminaries: Recovering the Mueller matrix}

Partially polarized light can be represented by means of its Stokes parameters, $S_{i}$ (with $i=0,1,2,3$ ) [9], which can be arranged to form a fourdimensional vector. The Stokes parameters, at any point in the space, can be related to a set of light intensities in the following way [6]:

$$
\mathbf{S}=\left[\begin{array}{c}
S_{0} \\
S_{1} \\
S_{2} \\
S_{3}
\end{array}\right]=\left[\begin{array}{c}
I_{0}+I_{\pi / 2} \\
I_{0}-I_{\pi / 2} \\
I_{\pi / 4}-I_{-\pi / 4} \\
I_{\lambda / 4, \pi / 4}-I_{\lambda / 4,-\pi / 4}
\end{array}\right]
$$

where $I_{\beta}$ and $I_{\lambda / 4, \beta}$ represent the intensity at that point after a linear polarizer and after a quarter-wave plate followed by a linear polarizer, respectively. The subscript $\beta$ refers to the angle formed by the transmission axis of the polarizer with the $x$ axis, while $\lambda / 4$ denotes the presence of the quarter-wave plate, having its fast axis at 0 . Owing to the above relations, the Stokes parameters are apparently quantities obtainable from intensity measurements. Specifically $S_{0}$ represents the irradiance of the beam at a point; $S_{1}$ is the difference between the amount of linearly polarized light at 0 and the amount of linearly polarized light at $\pi / 2 ; S_{2}$ is analogous to $S_{1}$ but considering linear polarization states at $\pi / 4$ and $-\pi / 4 ; S_{3}$ provides the difference of left and right circular polarization at such point.

When light passes through an optical system or sample, its polarization properties change. The output Stokes vector, $\mathbf{S}^{\text {out }}$, is related to the input Stokes vector, $\mathbf{S}^{\text {in }}$, through the $4 \times 4$ Mueller matrix, $\widehat{M}$, representing the polarization changes induced by the system:

$$
\mathbf{S}^{\text {out }}=\left[\begin{array}{cccc}
m_{00} & m_{01} & m_{02} & m_{03} \\
m_{10} & m_{11} & m_{12} & m_{13} \\
m_{20} & m_{21} & m_{22} & m_{23} \\
m_{30} & m_{31} & m_{32} & m_{33}
\end{array}\right] \mathbf{S}^{\text {in }}=\widehat{M} \mathbf{S}^{\text {in }}
$$

In general, in order to determine all the elements of the sample's Mueller matrix $\left(m_{i j}, i, j=0,1,2,3\right)$, at least 16 measurements have to be performed, involving the generation of four input polarization states, whose Stokes vectors must be linearly independent, and the projection of the output light 
onto four polarization states described by linearly independent Stokes vectors [4]. In some cases, however, for instance when samples present large linear retardance and negligible amounts of other polarization forms (such as some birefringent materials), a fewer number of measurements can be used, and the characterization of the sample turns out to be simpler. For example, in the three-polarization probing method [42, 43] three polarization states are used for probing the sample and only the projections onto three polarization states are measured. In this way, only a $3 \times 3$ submatrix of the Mueller matrix can be determined, but the latter is sometimes sufficient to recover the complete Mueller matrix. For instance, in the case of linear and deterministic samples, i.e. samples that can be represented by a Jones matrix [5, 41], the relations among the Mueller matrix elements allows for the complete Mueller matrix to be recovered from the knowledge of the above submatrix [43, 44].

Let us see in more detail the recovering process of the Mueller matrix with three probing polarization states, supposing that all of them are linear. With such an assumption, for any incident polarization state the fourth Stokes parameter vanishes and Eq. (2) can be rewritten as

$$
\mathbf{S}^{\text {out }}=\left[\begin{array}{c}
m_{00} S_{0}^{\text {in }}+m_{01} S_{1}^{\text {in }}+m_{02} S_{2}^{\text {in }} \\
m_{10} S_{0}^{\text {in }}+m_{11} S_{1}^{\text {in }}+m_{12} S_{2}^{\text {in }} \\
m_{20} S_{0}^{\text {in }}+m_{21} S_{1}^{\text {in }}+m_{22} S_{2}^{\text {in }} \\
m_{30} S_{0}^{\text {in }}+m_{31} S_{1}^{\text {in }}+m_{32} S_{2}^{\text {in }}
\end{array}\right] .
$$

If three different input linear polarization states are used, with linearly independent Stokes vectors $\mathbf{S}^{\text {in }, \ell}$ (with $\ell=1,2,3$ ), the measured intensities at the output are

$$
\left[\begin{array}{l}
S_{0}^{\text {out }, 1} \\
S_{0}^{\text {out }, 2} \\
S_{0}^{\text {out }, 3}
\end{array}\right]=\widehat{W}\left[\begin{array}{l}
m_{00} \\
m_{01} \\
m_{02}
\end{array}\right],
$$


130

with the $\widehat{W}$ matrix defined as

$$
\widehat{W}=\left[\begin{array}{ccc}
S_{0}^{\mathrm{in}, 1} & S_{1}^{\mathrm{in}, 1} & S_{2}^{\mathrm{in}, 1} \\
S_{0}^{\mathrm{in}, 2} & S_{1}^{\mathrm{in}, 2} & S_{2}^{\mathrm{in}, 2} \\
S_{0}^{\mathrm{in}, 3} & S_{1}^{\mathrm{in}, 3} & S_{2}^{\mathrm{in}, 3}
\end{array}\right]
$$

On inverting Eq. (4), the elements $m_{0 j}(j=0,1,2)$ are evaluated as

$$
\left[\begin{array}{c}
m_{00} \\
m_{01} \\
m_{02}
\end{array}\right]=\widehat{W}^{-1}\left[\begin{array}{c}
S_{0}^{\text {out }, 1} \\
S_{0}^{\text {out }, 2} \\
S_{0}^{\text {out }, 3}
\end{array}\right]
$$

Note that when a uniformly polarized light is used, the input beam polarization state must be changed from one measurement to the following one. But, if a nonuniformly polarized beam is used as input beam, different polarization states can be found at different points of the cross section of the beam.

The second and the third Stokes parameters of the output light can be obtained, for each of the incident polarization states, on measuring the output intensities $I_{0}, I_{\pi / 4}$, and $I_{\pi / 2}$. In fact, from Eq. (1) we have $S_{1}^{\text {out }}=I_{0}-I_{\pi / 2}$ and $S_{2}^{\text {out }}=I_{\pi / 4}-I_{-\pi / 4}=2 I_{\pi / 4}-I_{0}-I_{\pi / 2}$, where different decompositions of the total intensity $S_{0}^{\text {out }}=I_{0}+I_{\pi / 2}=I_{\pi / 4}+I_{-\pi / 4}$ have been taken into account [4]. Therefore, even the elements $m_{1 j}$ and $m_{2 j}(j=0,1,2)$ can be evaluated using the system in Eq. (4), with the same $\widehat{W}^{-1}$ matrix. In conclusion, we have

$$
\left[\begin{array}{c}
m_{i 0} \\
m_{i 1} \\
m_{i 2}
\end{array}\right]=\widehat{W}^{-1}\left[\begin{array}{c}
S_{i}^{\text {out }, 1} \\
S_{i}^{\text {out }, 2} \\
S_{i}^{\text {out }, 3}
\end{array}\right]
$$

145

with $i=0,1,2$, and the following $3 \times 3$ partial Mueller matrix

$$
\widehat{M}_{3 \times 3}=\left[\begin{array}{ccc}
m_{00} & m_{01} & m_{02} \\
m_{10} & m_{11} & m_{12} \\
m_{20} & m_{21} & m_{22}
\end{array}\right],
$$


is obtained. It should be noted that only one polarimetric measurement matrix $\widehat{W}$ is necessary for obtaining the three columns of the partial Mueller matrix.

The method proposed here for recovering the complete Mueller matrix takes advantage of a series of relations existing among the elements of the Mueller matrix of a deterministic sample [44]. In particular, 30 sums of 4 different combination of products of two matrix elements are equal to zero. More precisely, there are 12 quantities that must vanish, involving products of any two chosen rows or columns, and are of the form [44]

$$
\begin{array}{ccc}
a_{1}= & m_{00} m_{01}-m_{10} m_{11}-m_{20} m_{21}-m_{30} m_{31} \\
a_{2}= & m_{00} m_{02}-m_{10} m_{12}-m_{20} m_{22}-m_{30} m_{32} \\
\vdots & \vdots & \vdots \\
a_{12} & = & m_{20} m_{30}-m_{21} m_{31}-m_{22} m_{32}-m_{23} m_{33}
\end{array}
$$

On the other hand, there are 18 additional quantities, which involve sums or differences of subdeterminants of the Mueller matrix, that have to vanish as well. They can be written in the form [44]

$$
\begin{array}{ccc}
b_{1}= & m_{00} m_{11}-m_{10} m_{01}-m_{22} m_{33}+m_{23} m_{32} \\
b_{2}= & m_{02} m_{13}-m_{03} m_{12}+m_{20} m_{31}-m_{21} m_{30} \\
\vdots & \vdots & \vdots \\
b_{18} & = & m_{10} m_{21}-m_{11} m_{20}+m_{02} m_{33}-m_{03} m_{32}
\end{array}
$$

It can be noted that each of the above expressions of $a_{p}$ (with $p=$ 150 $1,2, \ldots, 12$ ) or $b_{q}$ (with $q=1,2, \ldots, 18$ ) involves a certain number of elements belonging to the last row or to the last column of the complete Mueller matrix. We denote such number by $L_{p}$ or $L_{q}^{\prime}$, respectively. Then the following cost function can be defined:

$$
C=\sum_{p=1}^{12} a_{p}^{2} / L_{p}+\sum_{q=1}^{18} b_{q}^{2} / L_{q}^{\prime} .
$$

In conclusion, after measuring the $M_{3 \times 3}$ partial Mueller matrix by means of the three-polarization probing method, an algorithm that searches for the minimum of the multivariable cost function of Eq. (11) can be implemented to obtained the seven unknown Mueller matrix elements $m_{3 j}$ and $m_{j 3}$ (with $j=0,1,2,3)$. 


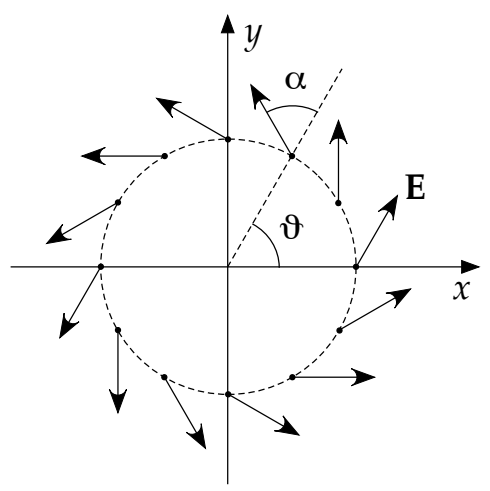

Figure 1: Distribution of polarization for a spirally polarized beam.

\section{Preliminaries: Spirally Polarized Beams}

We define a Spirally Polarized Beam (SPB) as a beam represented by the following Jones vector across a plane $z=0$ [37]:

$$
\mathbf{E}(\mathbf{r}, 0)=f_{0}(r)\left[\begin{array}{c}
\cos (\theta+\alpha) \\
\sin (\theta+\alpha)
\end{array}\right],
$$

160 plane and $\alpha$ is a constant angle. Since the polarization state is not defined
at the center of the profile, the function $f$ must be chosen in such a way that the amplitude vanishes at $r=0$.

The structure in Eq. (12) describes a field whose polarization is linear at SPB's own their name from the fact that the electric field lines are logarithmic spirals, whose growth parameter depends on the value of $\alpha$ [37]. On varying $\alpha$, different patterns of the polarization across the beam section are obtained, ranging from radial (when $\alpha=0$ ) to azimuthal (when $\alpha=\pi / 2$ ) polarization.

Using the Fresnel formula [45] to evaluate the propagated field in paraxial propagation, it is easily seen that, regardless the explicit form of the function $f_{0}$, the beam described in Eq. 12 keeps presenting the same polarization pattern across any transverse plane $z \neq 0$ [37, 38, 46, 47]. In fact, the expression of its Jones vector turns out to be of the form (12), but with the 
radial function replaced by the following

$$
f_{z}(r)=-\frac{k}{z} \mathrm{e}^{\mathrm{i} k z} \exp \left(\mathrm{i} \frac{k r^{2}}{2 z}\right) \int_{0}^{\infty} f_{0}(\rho) J_{1}\left(\frac{k r \rho}{z}\right) \exp \left(\mathrm{i} \frac{k \rho^{2}}{2 z}\right) \rho \mathrm{d} \rho,
$$

where $k$ is the wavenumber and $J_{1}$ the Bessel function of the first kind and order 1 [37. It is interesting to recall that the polarization pattern of the transverse electric field is propagation invariant even in nonparaxial conditions [40]. Closed expressions for the function $f_{z}(r)$ are obtained, for example, when the transverse function is of the Laguerre-Gaussian type [40. The behavior of SPB's focused by high-numerical-aperture systems was investigated in 48 .

The propagation-invariance property of the polarization pattern can be extended to all fields endowed with cylindrical symmetry, obtained by letting a SPB pass through a homogeneous deterministic optical element described by the Jones matrix $\widehat{J}$. The proof of this is very simple and stems from the linearity of the propagation operator and the homogeneity of the matrix $\widehat{J}$.

Let us introduce the direct propagator in free space, $K_{z}(\mathbf{r}, \boldsymbol{\rho})$, such that

$$
\mathbf{E}(\mathbf{r}, z)=\iint K_{z}(\mathbf{r}, \boldsymbol{\rho}) \mathbf{E}(\boldsymbol{\rho}, 0) \mathrm{d} \boldsymbol{\rho}=f_{z}(r)\left[\begin{array}{c}
\cos (\theta+\alpha) \\
\sin (\theta+\alpha)
\end{array}\right] .
$$

Equation (14) expresses the fact that, while the radial profile of the SPB changes on propagation according to Eq. (13), the polarization pattern keeps its initial form at any propagation distance.

If we let the SPB pass through the optical element characterized by the matrix $\widehat{J}$ and denote the emerging field by $\mathbf{E}^{\prime}$, we have

$$
\mathbf{E}^{\prime}(\mathbf{r}, 0)=\widehat{J} \mathbf{E}(\mathbf{r}, 0)=f_{0}(r) \widehat{J}\left[\begin{array}{c}
\cos (\theta+\alpha) \\
\sin (\theta+\alpha)
\end{array}\right]
$$

On studying the free propagation of the latter field, we have

$$
\begin{aligned}
\mathbf{E}^{\prime}(\mathbf{r}, z) & =\iint K_{z}(\mathbf{r}, \boldsymbol{\rho}) \mathbf{E}^{\prime}(\boldsymbol{\rho}, 0) \mathrm{d} \boldsymbol{\rho}=\iint K_{z}(\mathbf{r}, \boldsymbol{\rho}) \widehat{J} \mathbf{E}(\boldsymbol{\rho}, 0) \mathrm{d} \boldsymbol{\rho}= \\
& =\widehat{J} \iint K_{z}(\mathbf{r}, \boldsymbol{\rho}) \mathbf{E}(\boldsymbol{\rho}, 0) \mathrm{d} \boldsymbol{\rho}=\widehat{J} \mathbf{E}(\mathbf{r}, z)=f_{z}(r) \widehat{J}\left[\begin{array}{c}
\cos (\theta+\alpha) \\
\sin (\theta+\alpha)
\end{array}\right] .
\end{aligned}
$$


Equation (16), compared to Eq. (15), shows that even in this case the initial polarization pattern is preserved during propagation, the only effect of the latter being a modification of the radial profile. Note that the only condition required by Eq. (14) is that the function $f_{0}$ be dependent only on the radial distance $r$, so that any kind of radial profile can be considered, including circular apertures.

Of course the above property holds not only when $K_{z}$ is the direct propagator in free space, but for whatever linear scalar operator, and hence for the propagation of the output beam through any isotropic optical systems.

\section{Polarimetry with spirally polarized beams}

Using a SPB as the input beam, all possible linear polarization states are simultaneously present across the input plane of the sample. As recalled in Sec. 2, if the sample is homogeneous, linear and deterministic, three linear polarization states are sufficient to recover its complete Mueller matrix, so that the Stokes parameters have to be measured at three different points of the output plane. On the other hand, measuring the Stokes parameters at a larger number of points corresponds to considering a larger number of input polarization states. This can be used to reduce the experimental measurement variances.

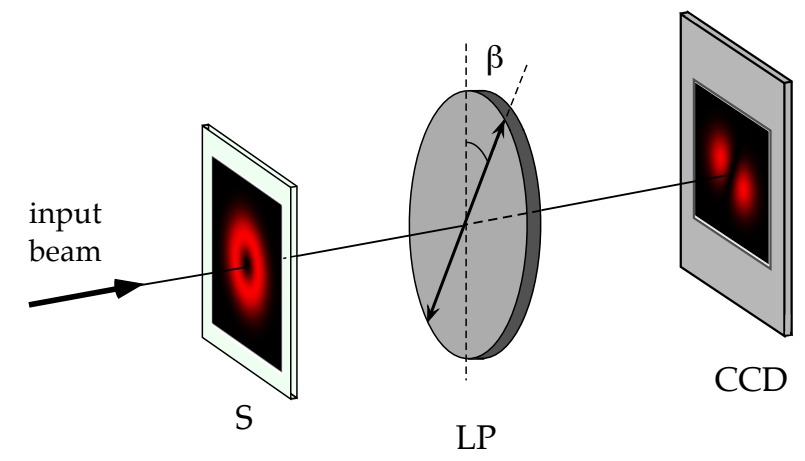

Figure 2: Schematic of the polarization analyzer.

Let us see in more detail the principle experimental scheme aimed at recovering the Mueller matrix of the sample (Fig. 2). The input beam is a SPB impinging onto the sample (S); a linear polarizer (LP) with adjustable direction of the transmission axis (identified by the angle $\beta$ ) is placed beyond 
the sample and acts as the analyzer; a CCD camera acquires the irradiance profile of the transmitted field.

According to Eq. (1), and recalling that $I_{\pi / 4}-I_{-\pi / 4}=2 I_{\pi / 4}-I_{0}-I_{\pi / 2}$, to measure the first three components of the Stokes vector of the field emerging from the sample, three images have to be acquired by the CCD, corresponding to three different angles of the polarizer $(\beta=0, \pi / 2, \pi / 4)$. Acquired images allows such components to be measured at any point of the output beam cross section. On selecting three of such points, the theory exposed in Sec. 2 allows the Mueller matrix of the sample to be derived. Since the polarization pattern of the input beam is invariant during propagation, the sample can be inserted at any transverse plane along its path. Furthermore, owing to the polarization invariance of the output beam, the field profile at the exit of the sample needn't to be imaged onto the CCD detector.

The approach presented in Sec. 2 can now be specialized to the case of an impinging SPB. The Stokes vector of a SPB is easily evaluated as [37, 38, 46]

$$
\mathbf{S}_{S P B}=I(r)\left[\begin{array}{c}
1 \\
\cos (2 \alpha+2 \theta) \\
\sin (2 \alpha+2 \theta) \\
0
\end{array}\right]
$$

where, for brevity, we denote the irradiance, $\left|f_{z}(r)\right|^{2}$, by $I(r)$, so that the Stokes vector of the output beam turns out to be

$$
\mathbf{S}^{\text {out }}(r, \theta)=I(r)\left[\begin{array}{l}
m_{00}+m_{01} \cos (2 \alpha+2 \theta)+m_{02} \sin (2 \alpha+2 \theta) \\
m_{10}+m_{11} \cos (2 \alpha+2 \theta)+m_{12} \sin (2 \alpha+2 \theta) \\
m_{20}+m_{21} \cos (2 \alpha+2 \theta)+m_{22} \sin (2 \alpha+2 \theta) \\
m_{30}+m_{31} \cos (2 \alpha+2 \theta)+m_{32} \sin (2 \alpha+2 \theta)
\end{array}\right],
$$

where the fact that $S_{3}^{\text {in }}(r, \theta)=0$ for a SPB has been taken into account.

By measuring the first three Stokes parameters at three points, namely $\left(r_{\ell}, \theta_{\ell}\right)$, with $\ell=1,2,3$, the nine elements of $\widehat{M}_{3 \times 3}$ are evaluated through the linear systems in Eq. (7). The involved matrix $\widehat{W}$, defined in Eq. (5), in this case takes the form

$$
\widehat{W}=\left[\begin{array}{lll}
I\left(r_{1}\right) & I\left(r_{1}\right) \cos \left(2 \alpha+2 \theta_{1}\right) & I\left(r_{1}\right) \sin \left(2 \alpha+2 \theta_{1}\right) \\
I\left(r_{2}\right) & I\left(r_{2}\right) \cos \left(2 \alpha+2 \theta_{2}\right) & I\left(r_{2}\right) \sin \left(2 \alpha+2 \theta_{2}\right) \\
I\left(r_{3}\right) & I\left(r_{3}\right) \cos \left(2 \alpha+2 \theta_{3}\right) & I\left(r_{3}\right) \sin \left(2 \alpha+2 \theta_{3}\right)
\end{array}\right] .
$$

However, since the input beam may differ from an ideal SPB, in a practical implementation of the method it is worthwhile to use measured values of the 
elements of the matrix $\widehat{W}$, directly obtained from the Stokes parameters of the input beam.

In order to exploit the large amount of information contained in the acquired images and to reduce the measurement errors, the Mueller matrix of the sample can be calculated from several sets of three points and the average of the obtained results can be obtained.

In the next section, we will evaluate the angles where to measure the Stokes vector, such that the Mueller matrix elements are recovered with the minimum error. Furthermore, we will present numerical simulations to evaluate the effects of several sources of error in a practical measurement.

\section{Angle optimization and error analysis}

Now we consider the effect of the errors in the polarimetric matrix $\widehat{W}$ (due to errors in the calibration of the input beam) and in the values of the measured output beam Stokes parameters on the recovered elements of the Mueller submatrix $\widehat{M}_{3 \times 3}$. The first three components of the Stokes vector of the output beam are measured at three points of the transverse plane, denoted by $\left(r_{\ell}, \theta_{\ell}\right)$, with $\ell=1,2,3$. We will derive the positions of such points, for which the error on the elements of $\widehat{M}_{3 \times 3}$ turns out to be minimum.

On denoting by $\Delta \widehat{W}$ the errors on $\widehat{W}$ and supposing that such errors, as well as the ones in measuring the output Stokes parameters, are small enough, the relative error in the determination of the elements of $\widehat{M}_{3 \times 3}$ is upper bounded by [42, 49]

$\delta\left(m_{i 0}, m_{i 1}, m_{i 2}\right)^{T} \leq \frac{\kappa(\widehat{W})\left(\delta \widehat{W}+\delta\left(S_{i}^{\text {out }, 1}, S_{i}^{\text {out }, 2}, S_{i}^{\text {out }, 3}\right)^{T}\right)}{1-\kappa(\widehat{W}) \delta \widehat{W}} ;(i=0,1,2),(20)$

where the superscript $T$ denotes the transpose, $\delta(\cdot)=\|\Delta(\cdot)\| /\|(\cdot)\|,\|(\cdot)\|$ is the Euclidean norm for vectors or the induced 2-norm for matrices, and $\kappa(\widehat{W})=\|\widehat{W}\| \cdot\left\|\widehat{W}^{-1}\right\|$ is the condition number of the polarimetric measurement matrix [10, 25, 42, 49].

It can be noted that the upper bound of the relative error $\delta\left(m_{i j}\right)$ grows linearly with the relative error of the output Stokes parameters, $\delta \mathbf{S}^{\text {out }, \ell}$, but that it increases linearly with $\delta \widehat{W}$ only when $\delta \widehat{W} \ll 1 / \kappa(\widehat{W})$. Minimization of $\kappa(\widehat{W})$, yields to the optimum set of the coordinates $\left(r_{\ell}, \theta_{\ell}\right)$ [10, 25, 42].

A source of errors is the assumption of using a perfectly spirally polarized input beam made in Section 2. When the polarization state is different than 
270 linear, $S_{3}^{\text {in }, \ell}$ is no longer zero and an error is added to the measured $S_{i}^{\text {out }, \ell}$. This added error is

$$
\Delta S_{i}^{\mathrm{out}, \ell}=m_{i 3} S_{3}^{\mathrm{in}, \ell} \leq m_{00} S_{3}^{\mathrm{in}, \ell} ; \quad(i=0,1,2)
$$

where the upper limit is given by the physical realizability conditions of Mueller matrices [6].

Due to the cylindrical symmetry of the input beam (both in irradiance 275 and in polarization), the distance $r$ from the beam axis does not affect to the polarization state, and the first position for measuring could be set as $\theta_{1}=0$ without loss of generality. Then, the minimization has to be done over the other two angles, $\theta_{2}$ and $\theta_{3}$, where the Stokes parameters have to be measured.

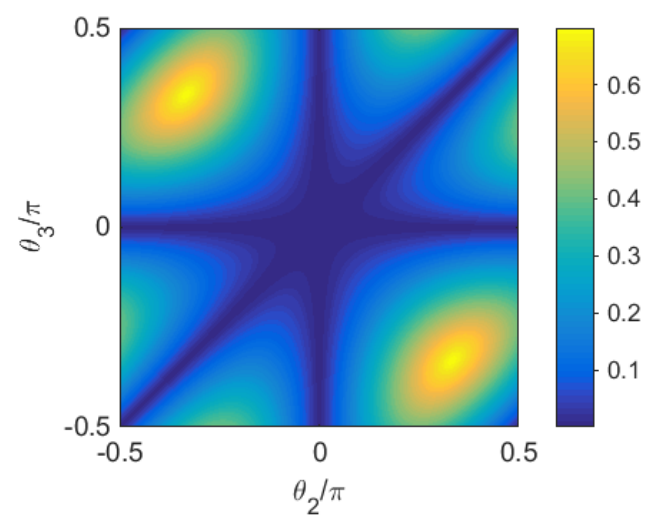

Figure 3: Inverse of the condition number $\kappa(\widehat{W})$ for all possible pairs of angles $\theta_{2}$ and $\theta_{3}$, being $\theta_{1}=0$.

Figure 3 shows a map of the inverse of $\kappa(\widehat{W})$ for all possible choices of the second and the third measurement directions, relative to the first one (2-norm). It can be noted that the optimum choices occur for $\theta_{2}= \pm \pi / 3$ and $\theta_{3}=\mp \pi / 3$, that is, when the three input linear polarization states form an equilateral triangle circumscribed into the equator of the Poincarè sphere [25, 42]. The minimum value of the condition number turns out to be $\simeq 1.42$. Although the minimum value for the condition number is different depending on the norm definition for matrices (1.42 for 2-norm, 3.73 for 1norm, 3.02 for $\infty$-norm, and 3.16 for Frobenius norm), the set of optimum measurement angles is the same for all considered definitions. As regards the 
optimum distance $r$ from the center of the beam, it is quite clear that the relative errors will be lower for points where the intensity is higher. Note that the results shown in Fig. 3 are independent of $\alpha$. This means that, regardless the value of $\alpha$, the optimum selection of the measurement positions will be located at three equally spaced angles, with $\pi / 3$ increment [42].

In the following of this section we present numerical simulations aimed at evaluating the effects of the presence of noise in practical measurements.

As already mentioned, the first source of noise is the fact that the experimental SPB used as the input beam generally differs from an ideal one, and discrepancies are expected due to nonzero values of its fourth Stokes parameter. To take this into account, we added pseudo-random noise to the input Stokes vector of an ideal SPB, at each point of the input plane. The maximum modulus of such noise is denoted by $N_{S}$.

The second considered source of error is the incorrect positioning of the polarization analyzer used to measure the Stokes parameters across the image plane. Therefore, a pseudo-random number, with modulus bounded by $N_{P}$, was added to the polarization angle of the polarizer.

Finally, the noise coming from acquisition by the camera is considered. In particular, a pseudo-random noise was added to the output image, with amplitude $N_{C}$, expressed as a fraction of the maximum recorded intensity. The quantization of the intensity values was also taken into account, for the case of a 8-bit AD conversion.

We emulated the complete measurement procedure, from the determination of the matrix $\widehat{W}$ to the recovering of the complete Mueller matrix of a sample, following the procedure described in Sections 2 and 4 , but including the above sources of noise at different stages of the process. The differences between theoretical and experimental Mueller matrix have been evaluated through the root mean square of the differences of the 16 Mueller matrix elements, that is,

$$
\epsilon=\sqrt{\frac{1}{16} \sum_{i, j=0}^{3}\left(m_{i, j}^{S}-m_{i, j}^{I}\right)^{2}},
$$

where $m_{i, j}^{S}$ are the elements of the Mueller matrix obtained from the simulation and $m_{i, j}^{I}$ are the elements of the Mueller matrix of the ideal sample. The process has been repeated several times and the mean value of $\epsilon$, namely, $\bar{\epsilon}$, has been evaluated.

Figure 4 shows the obtained values of $\bar{\epsilon}$ for the cases of samples consisting 
of a phase plate (for different retardances and orientations) and a diattenuator (for different diattenuations [6] and orientations). Used values of the errors are $N_{P}=\pi / 180$ for all cases and $N_{S}$ and $N_{C}$ varying from 2 to $10 \%$ of the maximum input beam intensity, depending on the example (see caption of Fig. 4). The number of different trials to obtain the average of $\epsilon$ is 20 .

It can be noted that the expected errors in the determination of the Mueller matrix are quite low for phase plates with almost any retardance value except when retardances are near to 0 and $\pi$ (see Fig. 4 a). This behaviour occurs when the fast axis is close to 0 and $\pi / 2$. The expected errors are higher for values of the retardance around $\pi / 4$ and $3 \pi / 4$ at different orientations of the fast axis (see Fig. $4 \mathrm{~b}$ ). In the case of linear diattenuators, the errors grow with the amplitude of the added noise and are slightly larger for high diattenuation than for low diattenuation values (see Fig. 4c). On the other hand these errors slightly grows with diattenuation for all orientations of the transmission axis but in any case, these errors are reasonably low (see Fig. 4.d)).

Finally, in order to take into account the combined effect of retardance and diattenuation, an additional simulation has been carried on (see Fig. 4.e). The sample has been simulated as a linear diattenuator followed by a phase plate, having both the transmission axis and the fast axis at 0 . The added noises correspond to $N_{P}=\pi / 180$ and $N_{S}=N_{C}=0.05$. It can be noticed that the expected errors in the determination of the Mueller matrix are quite low in most cases. The worst situation is for high values of the diattenuation and almost any retardance or when retardance is close to $\pi$ and diattenuation has a low value.

From the performed simulations it can be concluded that, in practical cases, Mueller matrix can be measured with reasonably accuracy if the errors in the calibration of the input beam and in the acquisition of the needed images are below $5 \%$ of the maximum input intensity.

\section{Experimental results and discussion}

To test the proposed method we used a SPB with $\alpha=\pi / 2$, i.e., an azimuthally polarized beam (see Fig. 1), as the incident beam, with the set up sketched in Fig. 5. Azimuthally polarized beams can be synthesized in several ways (see [39] and references therein), but in our experiment we used an Arcoptix liquid crystal polarization converter (PC) [38, 50]. 

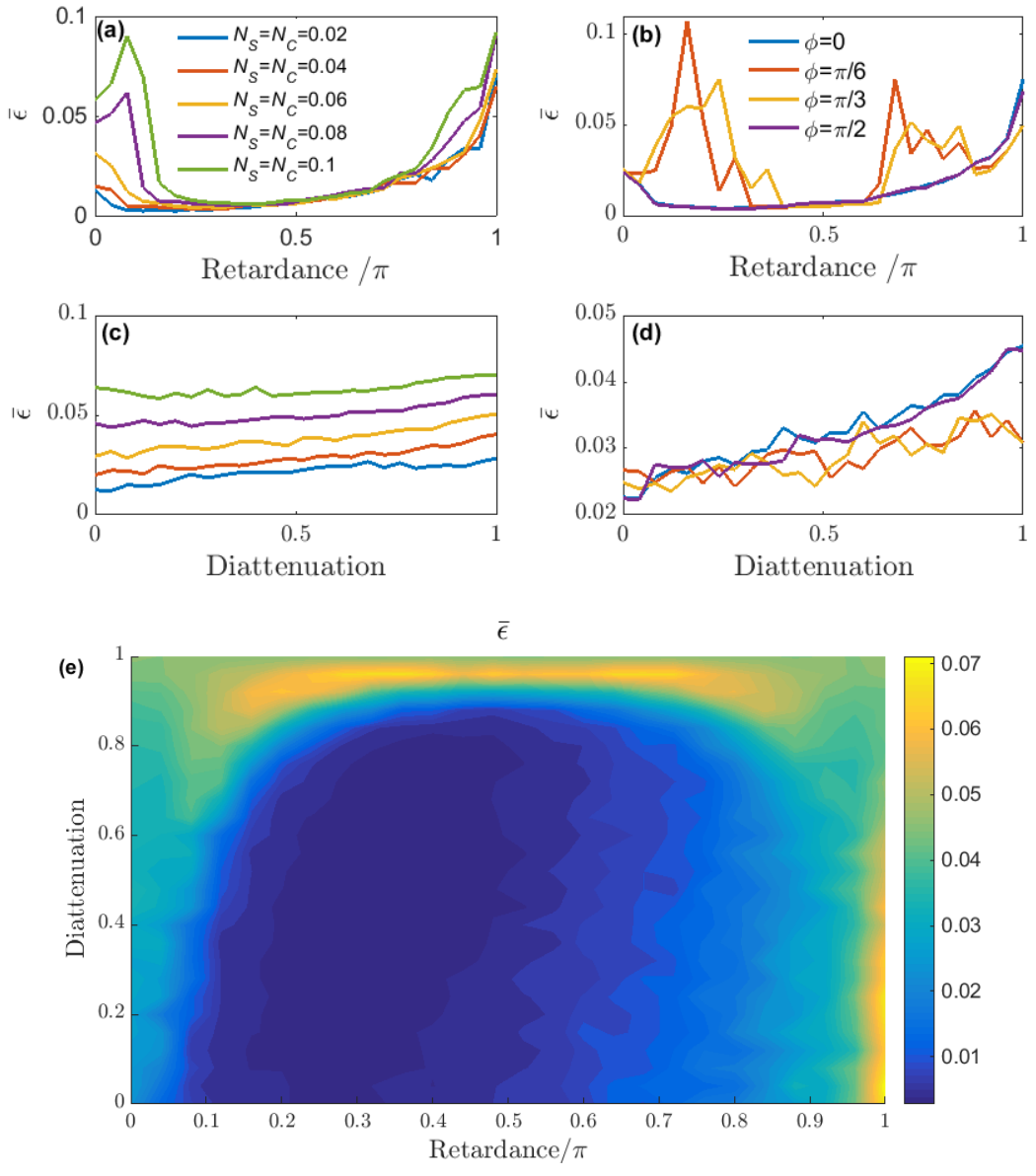

Figure 4: Average value of the root mean square of the difference between the calculated Mueller matrix and the ideal one for three different cases. 1) Phase plate with variable retardance and fast axis at 0 , for different values of the maximum amplitude of the added noise (a) and for different orientations of its axis with $N_{S}=N_{C}=0.05$ (b). 2) Linear diattenuator with variable diattenuation and transmission axis at 0 , for different values of the maximum amplitude of the added noise (c) (same levels as in (a)) and for different orientations the axis for $N_{S}=N_{C}=0.05$ (d) (same values as in (b)). 3) Combined retardance and diattenuation with $N_{S}=N_{C}=0.05$ (e). For all cases $N_{P}=\pi / 180$. 


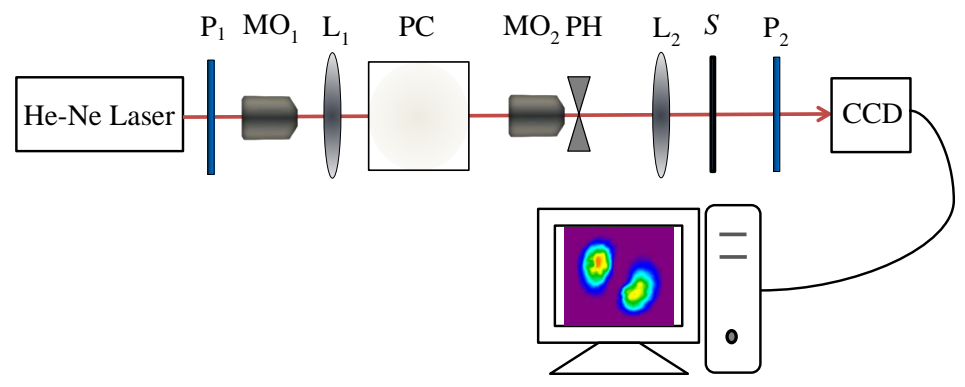

Figure 5: Experimental setup. $\mathrm{P}_{1}, \mathrm{P}_{2}$ : linear polarizers; $\mathrm{MO}_{1}, \mathrm{MO}_{2}$ : microscope objectives; $\mathrm{L}_{1}, \mathrm{~L}_{2}$ : lenses; PC Polarization converter; PH: pinhole; S: sample.

A He-Ne laser, stabilized both in intensity and in frequency, is used as the light source. A linear polarizer $\mathrm{P}_{1}$ selects the incident state of polarization, which must be linear with azimuth parallel to PC cell axis in order to obtain azimuthally polarized light at its exit [38]. The microscope objective $\mathrm{MO}_{1}$ and the lens $\mathrm{L}_{1}$ are used to expand the beam impinging onto the PC. After the $\mathrm{PC}$, the beam is spatially filtered and recollimated by means of a microscope objective, a pinhole, and a converging lens $\left(\mathrm{MO}_{2}, \mathrm{PH}\right.$, and $\mathrm{L}_{2}$, respectively). The filtered beam impinges onto the sample (S) and, after the linear polarizer $\mathrm{P}_{2}$ (which corresponds to the analyzer shown in Fig. 2), is sent onto the CCD camera (Pulnix TM-765).

A calibration of the polarization state across the beam section $\left(\right.$ after $\left.\mathrm{L}_{2}\right)$ was performed before using this beam as the probe for the sample characterization. To do this, the sample $S$ in Fig. 5 was removed and the Stokes parameters of the input beam were measured using the definitions in Eq. (1). It turned out that, as it was expected, such beam presents an intensity distribution with donut shape and is nearly azimuthally polarized, especially in the regions where the intensity is high. Furthermore, measurement of the input beam Stokes parameters allows us to select the points where $S_{3}$ is small enough, in order to reduce the error given by Eq. (21).

As homogeneous, deterministic and transparent samples we used a quarterwave phase plate (QWP), a half-wave phase plate (HWP) and a GlanThompson linear polarizer (GT) For each sample, two different orientations of the axes were used: fast axis or transmission axis along horizontal $(x)$ 375 direction and rotated by $-\pi / 6$. As was explained in Sec. 4, dealing with this kind of samples the measurement of the fourth component of the output beam Stokes vector is not necessary. On the other hand, the first three components 

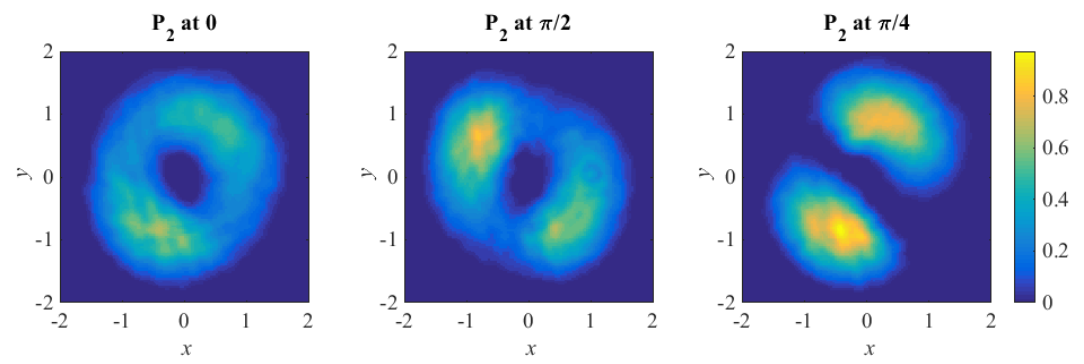

Figure 6: Experimental measured intensity (color scale in arbitrary units) across the beam section after the linear polarizer analyzer $\left(\mathrm{P}_{2}\right)$ with its transmission axis at $0, \pi / 2$, and $\pi / 4$, respectively, for a quarter-wave phase plate with its axes rotated by $-\pi / 6$.

can be measured without resorting to a quarter-wave plate (see Eq. (1)), so that three images have to be acquired by the camera, corresponding to three orientations of the linear polarizer $P_{2}(\beta=0, \pi / 2, \pi / 4$, with respect to the $x$ axis). As an example, Fig. 6 shows the acquired images of the output beam for the case of QWP with its axes rotated by $-\pi / 6$, for the three values of $\beta$. It can be observed that the images correspond to the intensity distribution that could be expected at the output of a rotated QWP for an azimuthally polarized input beam.

From the three experimental images, maps of the output Stokes parameters $S_{i}^{\text {out }}(r, \theta)$, with $i=0,1,2$, have been obtained. Several sets of three points have been used to evaluate the elements of the $\widehat{M}_{3 \times 3}$ submatrix through Eq. (7). According to the results recalled in Sec. 5, the polar angles of the three points in every set have been chosen as equally spaced by $\pi / 3$, at a distance from the beam axis where the input intensity was over the half of the maximum input intensity. From a set to the next one, the angles have been increased by $\pi / 90$ (e.g., $\theta_{1}$ ranged from $\pi / 90$ to $\pi / 3$, to avoid using of the same pixels, with step $\pi / 90$ ). Among all possible set of points, only those where $S_{3}^{\text {in }} \leq 0.05 S_{0}^{\text {in }}$ are considered. Finally, the mean value of each matrix element and its standard deviation have been calculated.

Table 1 collects the measured values of $M_{3 \times 3}$ for the QWP with the fast axis at 0 and $-\pi / 6$, together with the corresponding standard deviation. For comparison purposes, values of the elements are normalized to $m_{00}$ and this value is given separately. The same data are summarized in Table 2 for the HWP and in Table 3 for the linear polarizer. Note that $m_{00}$ gives the transmittance of the sample for unpolarized light [4, 5]. An independent measurement of this transmittance gave $m_{00}=0.95$ for the QWP, $m_{00}=0.98$ 
for the HWP and $m_{00}=0.45$ for the GT used as samples. Theoretical values corresponding to ideal elements are included for comparison. It can be noted a reasonable agreement between the experimental and theoretical values with discrepancies that are below 0.05. The maximum standard deviation is below 0.08 .

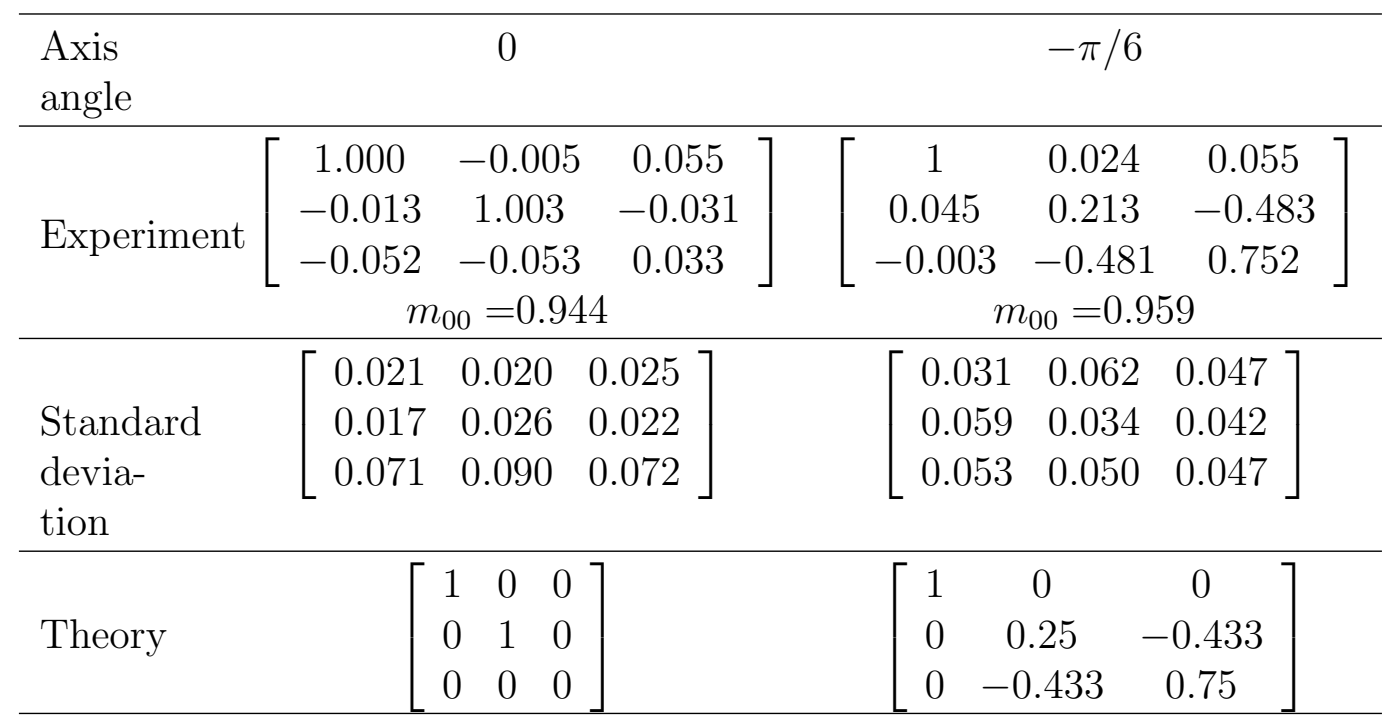

Table 1: Experimental values of the $\widehat{M}_{3 \times 3}$ partial Mueller matrix and standard deviation for a QWP with its axes rotated by a given angle with respect to the $x$ axis. Theoretical values are included.

As described in Sec. 2, the relations among the Mueller matrix elements for deterministic samples allows for the recovering of the complete Mueller matrix. Figure 7 shows the theoretical and experimental values of the normalized Mueller matrices obtained for the three considered samples, when their axes are set at 0 and $-\pi / 6$ with respect to the $x$ axis. A good agreement between theoretical values and experimental measurements is exhibited. The largest difference (about 0.09 for some elements of the forth row and column) occurs for the HWP. Others authors have proposed a different approach for recovering the complete Mueller matrix from the $\widehat{M}_{3 \times 3}$ submatrix [43]. They have shown that the errors in determining the retardance grows faster with the error in the measured $\widehat{M}_{3 \times 3}$ submatrix for sample retardance near to $\pi$, which is the case of a HWP, than for any other sample retardance. Similar results can be observed in Fig. 4.(a). This means that, even in the worst 


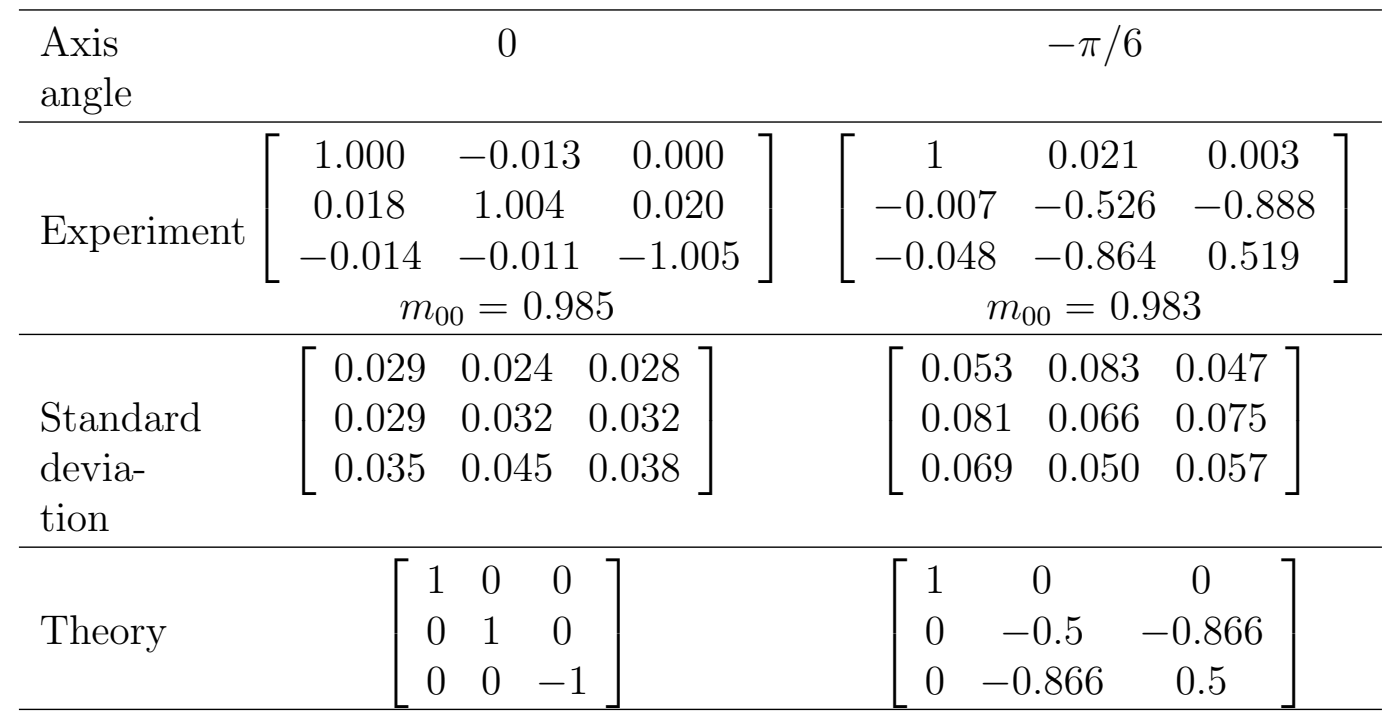

Table 2: Experimental values of the $\widehat{M}_{3 \times 3}$ partial Mueller matrix and standard deviation for a HWP with its axes rotated by a given angle with respect to the $x$ axis. Theoretical values are included.

case for recovering the complete Mueller matrix from the $\widehat{M}_{3 \times 3}$ submatrix, we obtain results with reasonably low values for the recovered elements.

\section{Conclusions}

A method to recover the Mueller matrix of homogeneous, linear and deterministic samples is proposed. The method is based on the use of SPB's as continuous polarization generators. SPB's give two main advantages: first, across any transverse plane, they present all possible linear polarization states; second, their transverse polarization pattern remains invariant under propagation, so that the sample can be placed at any transverse plane along the beam propagation axis. Furthermore, such an invariance property is preserved for the exiting beam when a SPB passes through a linear deterministic and homogeneous sample, so that the camera can be placed at any distance from the sample under test. Using a calibrated input SPB, only the measurement of the Stokes parameters $S_{0}, S_{1}$, and $S_{2}$ across the output beam cross section is necessary to obtain a $3 \times 3$ partial Mueller matrix. This can be carried out by means of a CCD camera, which has to take three images of 


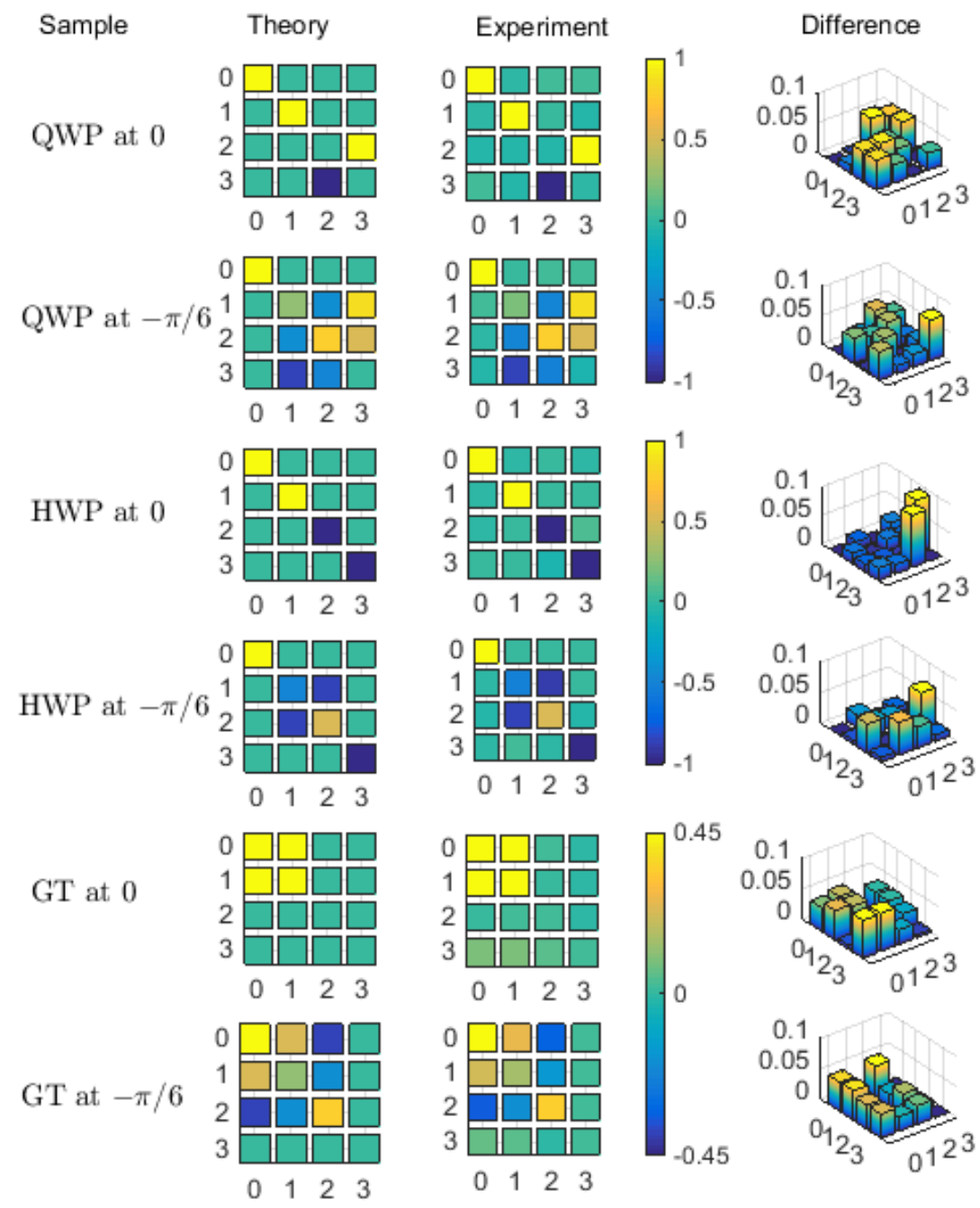

Figure 7: Theoretical and experimental Mueller matrix values, as well as corresponding absolute value of the difference among them for different samples. 


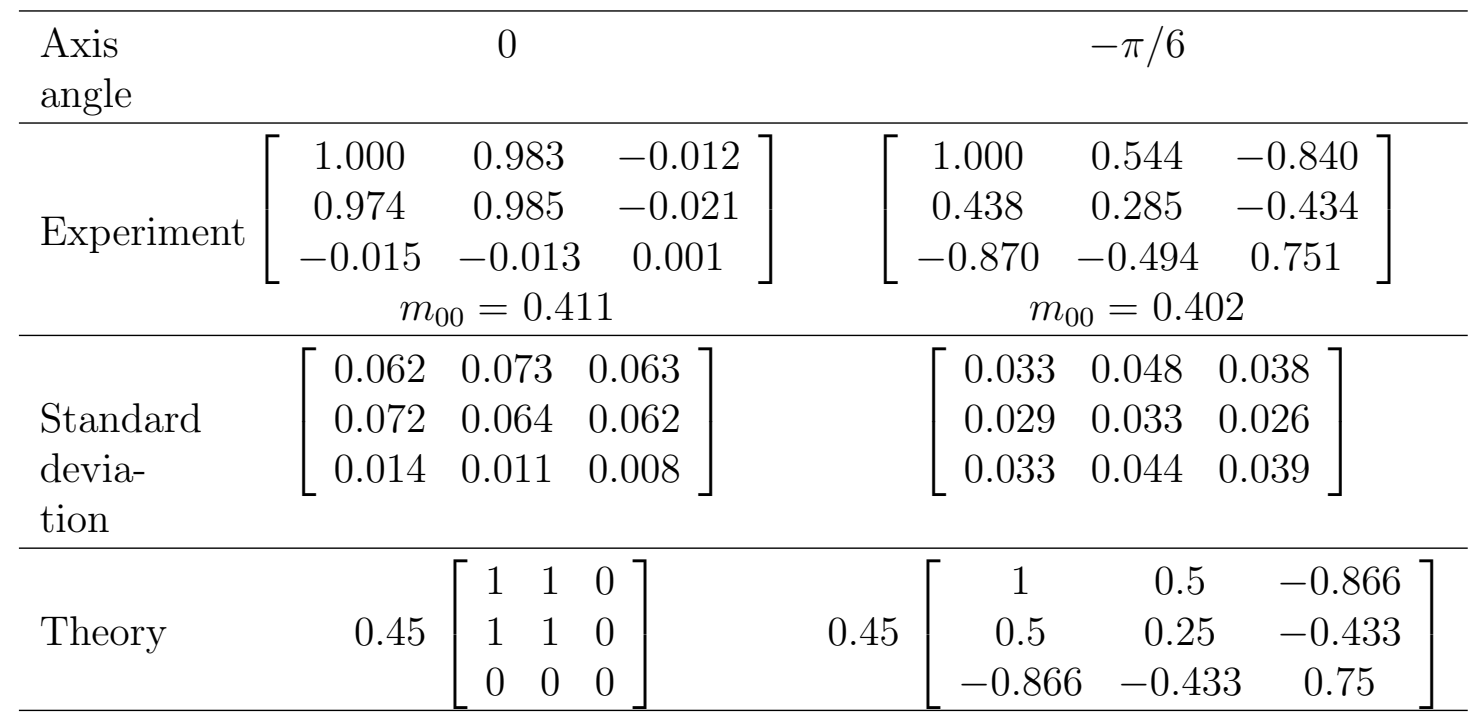

Table 3: Experimental values of the $\widehat{M}_{3 \times 3}$ partial Mueller matrix and standard deviation for a Glan-Thompson polarizer with its axes rotated by a given angle with respect to the $x$ axis. Theoretical values are included.

the output beam intensity, filtered by a suitably oriented analyzer. After obtaining the output Stokes parameters at three different points, chosen across the beam transverse section, a well conditioned system of linear equations can be written for the nine elements of the partial Mueller submatrix. Constraints on the Mueller matrix elements in the case of deterministic samples are exploited to recover the complete Mueller matrix. Numerical simulation and experimental results confirm the practical applicability of the proposed method.

Some improvements can be envisaged to enhance the performances of the method. For instance, to make the acquisition faster, an amplitude-division scheme could be used in the polarization analysis stage, as suggested in [26]. In that scheme, the output beam is divided into three identical replicas, which 450 are eventually analyzed by means of three linear polarizers with different transmission direction and recorded by three cameras, so that a single-shot Mueller polarimeter could be implemented. Alternatively, a single output image could be used, but filtered by a space varying polarizer. 


\section{Acknowledgments}

455 This work has been partially supported by Spanish Ministerio de Economía y Competitividad under project FIS2013-46475.

\section{References}

1. Tuchin VV, Wang L, Zymnayakov DA. Optical Polarization in Biomedical Applications. Springer; 2006. ISBN 9783540453215.

2. Wakaki M, ed. Optical Materials and Applications. CRC Press Taylor \& Francis Group; 2013.

3. Sujecki S. Photonics Modelling and Design. CRC Press Taylor \& Francis Group; 2015.

4. Goldstein DH. Polarized Light. Second (revised and expanded) ed.; Marcel Dekker, Inc.; 2003. ISBN 082474053X.

5. Gil JJ. Polarimetric characterization of light and media. The European Physical Journal Applied Physics 2007;40:1 - 47. URL: http://dx.doi. org/10.1051/epjap:2007153.

6. Chipman RA. Polarimetry; vol. I; chap. 15. McGraw-Hill Companies; third ed. ISBN 9780071598890; 2010:1 - 46.

7. de Sande JCG, Rodríguez A, Rodríguez T. Spectroscopic ellipsometry determination of the refractive index of strained $\mathrm{Si}_{1-x} \mathrm{Ge}_{x}$ layers in the near-infrared wavelength range (0.9-1.7 $\mu \mathrm{m})$. Applied Physics Letters 1995;67(23):3402-4. URL: http://scitation.aip.org/content/aip/ journal/apl/67/23/10.1063/1.114907, doi:http://dx.doi.org/10. 1063/1.114907.

8. de Sande JCG, Missana T, Afonso CN. Optical properties of pulsed laser deposited bismuth films. Journal of Applied Physics 1996;80(12):70237. URL: http://scitation.aip.org/content/aip/journal/jap/80/ 12/10.1063/1.363775, doi.http://dx.doi.org/10.1063/1.363775

9. Stokes GG. On the composition and resolution of streams of polarized light from different sources. In: Mathematical and Physical Papers; 
vol. 3. Cambridge University Press. ISBN 9780511702266; 2009:23358. URL: http://dx.doi.org/10.1017/CB09780511702266.010; cambridge Books Online.

10. Twietmeyer KM, Chipman RA. Optimization of Mueller matrix polarimeters in the presence of error sources. Opt Express 2008;16(15):11589-603. URL: http: //www.opticsexpress.org/ abstract. cfm?URI=oe-16-15-11589. doi:10.1364/0E .16.011589.

15. Mu T, Zhang C, Li Q, Liang R. Error analysis of single510

16. Tyo JS, Goldstein DL, Chenault DB, Shaw JA. Review of passive imaging polarimetry for remote sensing applications. Appl Opt 

ao-45-22-5453, doi:10.1364/A0.45.005453.

17. Peinado A, Turpín A, Lizana A, Fernández E, Mompart J, Campos J. Conical refraction as a tool for polarization metrology. Opt Lett 2013;38(20):4100-3. URL: http://ol.osa.org/abstract.cfm?URI= ol-38-20-4100, doi:10.1364/0L .38.004100.

18. Peinado A, Lizana A, Turpín A, Iemmi C, Kalkandjiev TK, Mompart J, Campos J. Optimization, tolerance analysis and implementation of a Stokes polarimeter based on the conical refraction phenomenon. Opt Express 2015;23(5):5636-52. URL: http://www.opticsexpress.org/ abstract. cfm?URI=oe-23-5-5636. doi:10.1364/0E. 23.005636.

19. Gorodetski Y, Biener G, Niv A, Kleiner V, Hasman E. Space-variant polarization manipulation for far-field polarimetry by use of subwavelength dielectric gratings. Opt Lett 2005;30(17):2245-7. URL: http://ol.osa. org/abstract. cfm?URI=ol-30-17-2245. doi:10.1364/0L . 30.002245.

20. Gori F. Measuring stokes parameters by means of a polarization grating. Opt Lett 1999;24(9):584-6. URL: http://ol.osa.org/abstract.cfm? URI=ol-24-9-584. doi:10.1364/0L . 24.000584.

21. Tripathi S, Toussaint KC. Rapid Mueller matrix polarimetry based on parallelized polarization state generation and detection. Opt Express 2009;17(24):21396-407. URL: http://www.opticsexpress.org/ abstract.cfm?URI=oe-17-24-21396. doi:10.1364/0E .17.021396.

22. Kenny F, Rodríguez O, Lara D, Dainty C. Vectorial polarimeter using an inhomogeneous polarization state generator. In: Frontiers in Optics 2011/Laser Science XXVII. Optical Society of America; 2011:FThQ5. URL: http://www.osapublishing.org/abstract.cfm? URI=FiO-2011-FThQ5, doi:10.1364/FI0.2011.FThQ5.

23. Martínez-Ponce G, Solano C, Pérez-Barrios C. Hybrid complete Mueller polarimeter based on phase modulators. Optics and Lasers in Engineering 2011;49(6):723 -8. URL: http://www.sciencedirect.com/ 545 science/article/pii/S0143816610002927. doi:http://dx.doi.org/ 10.1016/j.optlaseng.2010.12.011; research in Optics and Photonics at CIO, Mexico. 
24. Dev K, Asundi A. Mueller-Stokes polarimetric characterization of transmissive liquid crystal spatial light modulator. Optics and Lasers in Engineering 2012;50(4):599 - 607. URL: http://www.sciencedirect.com/ science/article/pii/S0143816611002934. doi:http://dx.doi.org/ 10.1016/j.optlaseng.2011.10.004; computational Optical Measurement.

25. Layden D, Wood MFG, Vitkin IA. Optimum selection of input polarization states in determining the sample Mueller matrix: a dual photoelastic polarimeter approach. Opt Express 2012;20(18):2046681. URL: http://www.opticsexpress.org/abstract.cfm?URI= oe-20-18-20466. doi:10.1364/0E.20.020466.

26. Töppel F, Aiello A, Marquardt C, Giacobino E, Leuchs G. Classical entanglement in polarization metrology. New Journal of Physics 2014;16(7):073019. URL: http://stacks.iop.org/1367-2630/16/i= $7 / \mathrm{a}=073019$.

27. Adhikari A, Dev K, Asundi A. Subwavelength metrological chracterization by mueller matrix polarimeter and finite difference time domain method. Optics and Lasers in Engineering 2016;86:242 7. URL: http://www.sciencedirect.com/science/article/pii/ S0143816616301208, doi:http://dx.doi.org/10.1016/j.optlaseng. 2016.06 .014 .

28. Arrizón V, Tepichin E, Ortiz-Gutierrez M, Lohmann A. Fresnel diffraction at $1 / 4$ of the Talbot distance of an anisotropic grating. Optics Communications 1996;127(4):171 -5. URL: http://www.sciencedirect. com/science/article/pii/0030401896001009. doi:http://dx.doi. org/10.1016/0030-4018(96)00100-9.

29. Tervo J, Turunen J. Transverse and longitudinal periodicities in 575 fields produced by polarization gratings. Optics Communications 2001;190(16):51 -7. URL: http://www.sciencedirect.com/science/ article/pii/S0030401801010938. doi/http://dx.doi.org/10.1016/ S0030-4018(01)01093-8.

30. Piquero G, Borghi R, Santarsiero M. Gaussian Schell-model beams propagating through polarization gratings. $J$ Opt Soc Am A 
2001;18(6):1399-405. URL: http://josaa.osa.org/abstract.cfm? URI=josaa-18-6-1399, doi:10.1364/JOSAA .18.001399.

31. Beckley AM, Brown TG, Alonso MA. Full Poincaré beams. Opt Express 2010;18(10):10777-85. URL: http://www.opticsexpress.org/ abstract. cfm?URI=oe-18-10-10777, doi:10.1364/OE.18.010777.

32. Martínez-Herrero R, Mejías P. Propagation and parametric characterization of the polarization structure of paraxial radially and azimuthally polarized beams. Optics and Laser Technology 2012;44(2):482 -5. URL: http://www.sciencedirect.com/science/article/pii/ S0030399211002465, doi:http://dx.doi.org/10.1016/j.optlastec. 2011.08.018.

33. Korotkova O, Wolf E. Changes in the state of polarization of a random electromagnetic beam on propagation. Optics Communications 2005;246(13):35 - 43. URL: http://www.sciencedirect.com/ science/article/pii/S0030401804011058, doi/http://dx.doi.org/ 10.1016/j.optcom.2004.10.078.

34. Gori F. Partially correlated sources with complete polarization. Opt Lett 2008;33(23):2818-20. URL: http://ol.osa.org/abstract.cfm? URI=0l-33-23-2818, doi $10.1364 / 0 \mathrm{~L} .33 .002818$.

35. de Sande JCG, Santarsiero M, Piquero G, Gori F. Longitudinal polarization periodicity of unpolarized light passing through a double wedge depolarizer. Opt Express 2012;20(25):27348-

1 60. URL: http://www.opticsexpress.org/abstract.cfm?URI= oe-20-25-27348, doi:10.1364/OE.20.027348.

36. Santarsiero M, de Sande JCG, Piquero G, Gori F. Coherencepolarization properties of fields radiated from transversely periodic electromagnetic sources. Journal of Optics 2013;15(5):055701. URL: http://stacks. iop.org/2040-8986/15/i=5/a=055701.

37. Gori F. Polarization basis for vortex beams. J Opt Soc Am A 610 a 2001;18(7):1612-7. URL: http://josaa.osa.org/abstract.cfm?URI= josaa-18-7-1612. doi:10.1364/JOSAA.18.001612.

38. Ramírez-Sánchez V, Piquero G, Santarsiero M. Generation and characterization of spirally polarized fields. Journal of Optics A: Pure 
and Applied Optics 2009;11(8):085708. URL: http://stacks .iop.org/ $1464-4258 / 11 / i=8 / a=085708$.

39. Zhan Q. Cylindrical vector beams: from mathematical concepts to apa plications. Adv Opt Photon 2009;1(1):1-57. URL: http://aop.osa. org/abstract. cfm?URI=aop-1-1-1. doi:10.1364/AOP .1.000001.

40. Borghi R, Santarsiero M. Nonparaxial propagation of spirally po620 larized optical beams. J Opt Soc Am A 2004;21(10):2029-37. URL: http://josaa.osa.org/abstract.cfm?URI=josaa-21-10-2029. doi:10.1364/JOSAA.21.002029.

41. Simon R. Nondepolarizing systems and degree of polarization. Optics Communications 1990;77(5):349 -54. URL: http://www. sciencedirect.com/science/article/pii/003040189090123B. doi:http://dx.doi.org/10.1016/0030-4018(90)90123-B.

42. Oberemok EA, Savenkov SN. Determination of the polarization characteristics of objects by the method of three probing polarizations. Journal

1 of Applied Spectroscopy 2002;69(1):72-7. URL: http://dx.doi.org/ 10.1023/A:1015363709617, doi:10.1023/A:1015363709617.

43. Swami M, Patel H, Gupta P. Conversion of $3 \times 3$ Mueller matrix to $4 \times 4$ Mueller matrix for non-depolarizing samples. Optics Communications 2013;286:18 - 22. URL: http://www.sciencedirect.com/science/ article/pii/S003040181200942X. doi/http://dx.doi.org/10.1016/ j.optcom.2012.08.094.

44. Hovenier JW. Structure of a general pure Mueller matrix. Appl Opt 1994;33(36):8318-24. URL: http://ao.osa.org/abstract.cfm?URI= ao-33-36-8318, doi $10.1364 / \mathrm{AD} .33 .008318$.

45. Born M, Wolf E. Principles of Optics. Sixth (corrected) ed.; Cambridge University Press; 1980. ISBN 0521639212.

46. Martínez-Herrero R, Mejías PM, Piquero G. Characterization of Partially Polarized Light Fields. Springer Series in Optical Science; Springer; 2009. ISBN 9783642013270. 
47. Wu G, Wang F, Cai Y. Coherence and polarization properties of 645 a radially polarized beam with variable spatial coherence. Opt Express 2012;20(27):28301-18. URL: http://www.opticsexpress.org/ abstract. cfm?URI=oe-20-27-28301. doi:10.1364/0E .20.028301.

48. Borghi R, Santarsiero M, Alonso MA. Highly focused spirally polarized beams. J Opt Soc Am A 2005;22(7):1420-31. URL: http://josaa. osa.org/abstract.cfm?URI=josaa-22-7-1420. doi:10.1364/JOSAA . 22.001420.

49. Zallat J, S A, Stoll MP. Optimal configurations for imaging polarimeters: impact of image noise and systematic errors. Journal of Optics 655 A: Pure and Applied Optics 2006;8(9):807. URL: http://stacks.iop. org $/ 1464-4258 / 8 / i=9 / a=015$.

50. Ramírez-Sánchez V, Piquero G, Santarsiero M. Synthesis and characterization of partially coherent beams with propagationinvariant transverse polarization pattern. Optics Communications 2010;283(22):4484 -9. URL: http://www.sciencedirect.com/ 660 science/article/pii/S0030401810004220. doi:http://dx.doi.org/ 10.1016/j.optcom.2010.04.081; electromagnetic Coherence and Polarization. 